\title{
Quality of HIV Counselling Services Offered in Public Health Facilities in Kampala, Uganda
}

\section{Victo Kyobutungi ${ }^{1 *}$, Anthony Ssebagereka ${ }^{1}$, Christine T Begumisa ${ }^{1}$, Christine Muhumuza ${ }^{1}$ and Joseph KB Matovu ${ }^{2}$}

${ }^{1}$ Department of Epidemiology and Biostatistics, Makerere University College of Health Sciences, School of Public Health, P.O Box 7072, Kampala, Uganda

${ }^{2}$ Department of Community Health and Behavioral Sciences, Makerere University College of Health Sciences, School of Public Health, P.O Box 7072, Kampala, Uganda

*Corresponding Author: Victo Kyobutungi, Department of Epidemiology and Biostatistics, Makerere University College of Health Sciences, School of Public Health, P.O Box 7072, Kampala, Uganda.
Received: December 22, 2021

Published: February 25, 2022

(C) All rights are reserved by Victo

Kyobutungi., et al.

\section{Abstract}

Background: HIV counselling is increasingly available in public health facilities in Uganda. Counselling is an entry point into care, treatment and support services of many HIV/AIDS prevention programs. Quality of HCT services have significant role on prognosis of HIV patients. This study assessed the quality of HIV counselling services offered in public health facilities in Kampala by reviewing adherence to the HIV Counselling and Testing (HCT) policy guidelines, content of counselling information offered to clients and health facility amenities.

Methods: This was a cross-sectional study conducted in 2016 utilizing quantitative methods of data collection. Quality of HIV counseling was defined in accordance with adherence to the HCT guidelines, offering 'good' content of counselling information and health facilities having adequate amenities. We used principal component analysis to obtain and reveal current composite scores of the variables. Data were summarized into frequencies and proportions using STATA, version 13.0. Scores below 70\% were considered as 'poor' quality.

Results: In this study, 74 health workers offering HIV counselling services at five public health facilities were interviewed. The study revealed that quality of HIV counselling at the five public health facilities was 'poor' as 74\% of the health workers offered 'poor' quality HIV counselling and only one out of five health facilities was found to have adequate amenities. Majority (74.3\%) of the health workers were found not to adhere to HCT guidelines. In addition, $67.6 \%$ of the health workers offered 'poor' content of counseling information.

Conclusion: Overall quality of HIV counselling was found to be poor. Improvements should be focused on mentorship programs for all health workers involved in HIV counselling. Provision of modest space for counselling is also required so as to ensure privacy during counselling sessions.

Keywords: HCT: HIV Counselling and Testing; PCA: Principal Component Analysis 


\section{Introduction}

Worldwide, counselling in HIV is a gate way to comprehensive HIV care and support [1] and is widely accepted as the cornerstone of HIV prevention programs in many countries. In Sub-Saharan Africa, access to ART treatment continues to scale up which should stimulate innovative strategies to increase access to HCT services [2]. In this study quality of HIV counselling was defined in accordance with adherence to the HCT guidelines, offering 'good' content of counselling information and health facilities having adequate amenities.

Counselling in HIV has demonstrated multiple public health benefits [3] such as knowing one's HIV status, reduction in risky sexual behavior [4], linking HIV infected individuals to HIV care for support and treatment [5,6] among others. In addition, studies carried out in sub Saharan Africa including Uganda have shown that HIV counselling increases uptake of testing by $80 \%$ [7-12].

However, other authors have cited uptake of HIV counselling and testing to be low [13-15]. This is as a result of barriers to HIV counselling and testing which include substandard quality of counselling services, disclosure of HIV-positive status to an HIV-negative spouse that could result in abandonment, divorce or physical abuse against the woman [16], stigma, fear of knowing ones HIV status and blame [17-19]. A study conducted by Zachariah., et al. established that poor supervision affected the quality of counselling provided by health workers [20]. Similarly, a study conducted in Kenya reported that window period, partner involvement and follow-up support were haphazardly dealt with during HIV counselling [21].

Various studies in other countries have established that insufficient number and availability of trained counselors as well as lack of knowledge and competences by the counselors [22] were the biggest challenges affecting the quality of HIV counselling services [23]. The magnitude of these aspects and the quality of HIV counselling in Uganda is, however, minimally documented. In addition, there's insufficient information (especially in Uganda) on process related to HIV counselling quality indicators [23,24]. Studies assessing adherence to the HCT policy guidelines using the health systems perspective, evaluating the content of counselling information offered to clients during pre and post-test counselling sessions and assessing the health facility amenities that enhance quality of HIV counselling in public health facilities are warranted.
The objective of this study was to assess the quality of HIV counselling services offered in public health facilities in Kampala, Uganda.

\section{Methods}

Study setting and design

This was a cross-sectional study employing quantitative methods of data collection. Face-to-face interviews with counsellors and health workers providing counselling services to clients at five public health facilities (one Health Centre IV and four Health Centre IIIs) in Kampala were conducted. In Uganda, the healthcare system at subnational level is structured along the major levels of administration. A health center III is a facility having a catchment geographical area of a sub-county/division. Health center IIIs often have about 18 staff, led by a senior clinical officer, who runs a general outpatient clinic and a maternity ward. They also have a functioning laboratory.

On the other hand, Health center IV's serve a catchment population/geographical area of a county or a parliamentary constituency. Health center IVs offer all the services found at health center III, but also have inpatient wards for men, women, and children. It has a senior medical officer, medical officers, nurses, midwives, laboratory technicians and laboratory assistants. Health center IVs also have theatre facilities for carrying out major surgery procedures if required. Respondents who consented to take part in the study were included while those that were on leave or sick were excluded from the study.

A sample size of 74 respondents was determined using the Yamane formula at $95 \%$ confidence level. Kampala capital city is composed of 5 divisions and since the scope of the study was Kampala city, we decided to sample one health facility per division. Each division had to be represented in our sample of health facilities so that the findings would be representative of the city. And each division contains one health center III and one health center IV which are operated by the government of Uganda. Therefore, one functional health facility from each division was purposively selected. In addition, 12 and 26 respondents from health center III's and IV's respectively were proportionately sampled. Data collection tools were adapted from a study conducted by Mayanja [25] and modified following the national HCT guidelines to suit the study objectives. In addition, information from the standardized UNAIDS tool for assessing quality of HIV counselling was also used to modify the 
questionnaire [26]. The questionnaire was pre-tested to ensure its reliability and validity.

Data collection procedures and methods

Face to face interviews with HIV counselling service providers were done. Quantitative data on adherence to the Ministry of Health counselling guidelines, pre-test counseling, post-test counselling and health facility amenities was collected. The amenities deal with the extent to which the health facility's physical infrastructure is welcoming and pleasant such as clean surroundings, adequate furniture, sufficient ventilation, clean running water, and clean toilets among others.

\section{Variables and measurement}

The dependent variable was quality of HIV counselling that was measured in two ways; that is, self-assessment, and analysis of the dimensions of quality of HIV counselling services. On self-assessment, service providers were asked to rate the quality of HIV counselling they offered (based on the study's operational definition of HIV counselling) using a five level Likert scale of 'very good', 'good', 'fair', 'poor' and 'very poor'. The Independent variables included content of counselling information offered during pre and posttest counselling, adherence to HCT guidelines and facility amenities. Quality of HIV counselling was then collapsed into a binary outcome: that is, 'very good' and 'good' responses were combined into the 'good' category while 'fair', 'poor' and 'very poor' were also combined in the 'poor' category.

\section{Data analysis}

Descriptive statistics inform of frequencies and proportions were used to summarise the data. Principal Component Analysis (PCA) was used for analysis. All variables were prepared to measure the different components used to measure quality of HIV counselling in the same direction. Pairwise correlation between the variables was assessed with many variables scoring a correlation of above 0.3 which made it possible to proceed with PCA. Bartlett test of sphericity which compares the correlation matrix with a matrix of zero correlations was also carried out producing a statistically significant $P$ value $(p<0.001)$. Extraction of variables for PCA for the different dimensions (adherence to HCT guidelines, content of counselling information and amenities) followed by assessing Cronbach's Alpha were done. Variables measuring content of counselling information (both pre- and post-test counselling) were combined since they measured the same component. Variables that were collinear were removed as they could not produce valid factor loadings. Variables with low factor loadings were dropped until a desirable Cronbach's Alpha was attained. The final set of variables for each dimension were selected and used to create solid composite scores that were used to quantify the dimension's score. All frequencies from the three dimensions, used to assess quality of HIV counselling, were then combined and divided into textiles to obtain an overall measure of quality of HIV counselling. The cut off score used for grading the quality of HIV counselling was $70 \%$, where the score below that was categorized as 'poor' and the score above 70\% was rated 'good' [27,28]. Likewise, the same cut off was utilised for the scores in the three dimensions used to assess the quality of HIV counselling. Data were analysed using STATA version 13.0.

\section{Ethical considerations}

Ethical approval and clearance for the study was sought from the Makerere University School of Public Health Higher Degrees, Research and Ethics Committee, Kampala Capital City Authority and the health facilities' administrators. Written consent was also sought from the respondents.

\section{Results}

Overall 74 HIV counselling service providers were interviewed with a response rate of $100 \%$. Registered midwives comprised the highest number of HIV counselling service providers with 47 $(63.5 \%)$. Majority of the counselling service providers were female constituting $91.9 \%$ with a mean age of $36 \pm 7$ years. The experience in HIV counselling among HIV counselling service providers widely varied with majority (66.2\%) of them possessing an experience of above two years. Majority of the respondents (66.2\%) reported that there was compromised confidentiality during counselling sessions while $13.5 \%$ had not received any form of training in HIV counseling. Linkage into care of all the HIV positive clients at the ART clinic or ANC entry point was found to be $100 \%$. Also most counselling service providers 49 (66.2\%) did not follow $\mathrm{MOH}$ counselling guidelines while 51 (68.9\%) of reported absence of private space for counselling. Partner organizations constituted the highest proportion (67.6\%) in training of health workers and Infectious Diseases Institute (IDI) played a big role in support supervision as compared to Ministry of Health (MOH) and Kampala Capital City Authority (KCCA). 
Pre-test counselling

All (100\%) HIV counselling service providers revealed information concerning knowledge about HIV and its transmission to the client. The HIV testing process was discussed by $59.5 \%$ counselling service providers, 19 (25.7\%) discussed the window period while $43.2 \%$ discussed the value of partner involvement. Only 26 (35.1\%) of the counselling service providers talked about implications of a positive result for the baby and future pregnancies while only 12 $(16.2 \%)$ revealed that they assess for understanding of information passed on to the clients. Details are shown in table 1 below.

\begin{tabular}{|l|c|c|}
\hline Pre-test counseling (n= 74) & $\mathbf{N}$ & $\mathbf{\%}$ \\
\hline Knowledge about HIV and transmission & 74 & 100 \\
\hline HIV testing process & 44 & 59.5 \\
\hline Window period & 19 & 25.7 \\
\hline $\begin{array}{l}\text { Meaning and possible implications of HIV + and } \\
\text { HIV-negative results }\end{array}$ & 41 & 55.4 \\
\hline Partner involvement & 32 & 43.2 \\
\hline Potential needs and available support & 33 & 44.6 \\
\hline Transmission risk to the infant & 61 & 82.4 \\
\hline Benefits of knowing HIV status & 50 & 67.6 \\
\hline $\begin{array}{l}\text { Implications of a positive result for the baby and } \\
\text { future pregnancies }\end{array}$ & 26 & 35.1 \\
\hline Check for understanding & 12 & 16.2 \\
\hline
\end{tabular}

Table 1: Assessment of content of pre-test counselling.

Post-test counselling

We also found out that $91.9 \%$ of the HIV counselling service providers delivered results to clients in a clear and simple manner while $63.5 \%$ of the counselling service providers checked for the clients understanding to ensure that they had understood all the information they had passed on to them. Only $29.7 \%$ mentioned the need of disclosure, $24.3 \%$ of counselling service providers talked about the importance of family planning while $27 \%$ of the HIV counselling service providers scheduled next appointments with the clients for review as detailed in table 2 .

\section{Amenities}

All five health facilities had a waiting area where clients sat as they waited for counselling services and all the facilities had HCT policy manuals though they were locked up in cupboards. Information, education and communication materials were available in three of the health facilities. Whereas all the facilities had sufficient ventilation, privacy curtains were only observed in three of the examination rooms. Of the five health facilities observed, a private counselling room was available in only one health facility and only two of the health facilities had adequate furniture for their clients. Two of the health facilities had clean washrooms, and only one facility had a resting room for their clients. On self-assessment, 57\% HIV counselling service providers rated the quality of HIV counselling offered to clients as 'poor'.

\begin{tabular}{|l|c|c|}
\hline Post-test counselling (n=74) & N & $\mathbf{\%}$ \\
\hline Results given simply and clearly & 68 & 91.9 \\
\hline Checking for understanding & 47 & 63.5 \\
\hline Discussion of the meaning of the result for the client & 48 & 64.9 \\
\hline $\begin{array}{l}\text { Discussion of personal, family and social implica- } \\
\text { tions }\end{array}$ & 24 & 32.4 \\
\hline Disclosure & 22 & 29.7 \\
\hline Managing immediate emotional reactions & 27 & 36.5 \\
\hline Explanation of the delivery processes & 25 & 33.8 \\
\hline Family planning & 18 & 24.3 \\
\hline Previous ARV use & 25 & 33.8 \\
\hline Explanation of the ARV regimen and its role & 59 & 79.7 \\
\hline Adherence to treatment & 57 & 77 \\
\hline Next appointment made & 20 & 27 \\
\hline
\end{tabular}

Table 2: Assessment of content of post-test counselling.

Seven variables used to assess availability of health facility amenities exhibited a higher Cronbach's Alpha of 0.6536. These included availability of a counselling room, adequate furniture for clients, and availability of rest rooms, reading materials, privacy curtains in examination rooms, sufficient ventilation and clean washrooms. Only one out of the five heath facilities had adequate amenities as showed in table 3 below.

\begin{tabular}{|l|c|c|}
\hline $\begin{array}{l}\text { Tertiles for } \\
\text { amenities }\end{array}$ & $\begin{array}{c}\text { Frequency (Number } \\
\text { of Health facility) }\end{array}$ & Percentage \\
\hline 1 & 2 & 40 \\
\hline 2 & 2 & 40 \\
\hline 3 & 1 & 20 \\
\hline
\end{tabular}

Table 3: Tertiles for the measures of amenities. 
Tertiles are any two points that divide an ordered distribution into three equal parts, each containing a third of the population. These were used to generate a composite measure for quality of counselling. Tertiles were generated by divided the data equally into three portions and utilizing the $70 \%$ cutoff, the level of each composite variable and in turn the overall quality of counselling was determined.

\section{Adherence to HCT guidelines}

Results from the Principal Component Analysis (PCA) suggest that adherence to HCT guidelines is influenced by receipt of training in HIV counselling from a reputable trainer, following counselling guidelines, availability of private space for counselling, seeking consent from the clients and linkage into care when found positive. These variables produced a Cronbach's Alpha of 0.5172. Utilizing the $70 \%$ cut off, only $25.7 \%$ of the counselling service providers adhered to the HCT guidelines as detailed in table 4 below.

\begin{tabular}{|l|c|c|}
\hline $\begin{array}{l}\text { Tertiles of } \\
\text { Adherence }\end{array}$ & Frequency & Percentage \\
\hline 1 & 29 & 39.2 \\
\hline 2 & 26 & 35.1 \\
\hline 3 & 19 & 25.7 \\
\hline
\end{tabular}

Table 4: Tertiles for the measures of adherence to HCT guidelines.

\section{Content of counselling information}

Variables found to measure content of counselling information included: sharing of information on topics such as 'window period', partner involvement, HIV transmission risk to the infant, benefits of knowing one's HIV status, delivery of results in a clear and simple way, disclosure to the partner, explanation of the delivery process to the client, family planning, seeking past history of ARV use, explaining the ARV regimen and its role, adherence to treatment, making the next appointment with the client and checking to see if the client had understood all the information. These variables (shown in table 5) produced an inter-item correlation with a Cronbach's Alpha of 0.6531 . Since the third tertile lies above the 70\% cutoff, therefore only $32.4 \%$ of the HIV counselling service providers offered good content of counselling information.

\section{Overall quality of HIV counselling}

Average percentage score for the three dimensions of quality was used to obtain the overall quality of HIV counsellling, as de- tailed in table 6 below. Thus, only $26 \%$ of the HIV counselling service providers offered 'good' quality of HIV counselling.

\begin{tabular}{|l|c|c|}
\hline $\begin{array}{l}\text { Tertiles for content of } \\
\text { counselling information }\end{array}$ & Frequency & Percentage \\
\hline 1 & 25 & 33.8 \\
\hline 2 & 25 & 33.8 \\
\hline 3 & 24 & 32.4 \\
\hline
\end{tabular}

Table 5: Tertiles for the measures of content of counselling information.

\begin{tabular}{|l|c|}
\hline $\begin{array}{l}\text { Tertiles for quality of } \\
\text { HIV counselling }\end{array}$ & Percentage \\
\hline 1 & 37.7 \\
\hline 2 & 36.3 \\
\hline 3 & 26.0 \\
\hline
\end{tabular}

Table 6: Overall quality of HIV counselling services.

\section{Discussion}

Our findings on the quality of counseling services provided in public health facilities in Kampala, Uganda, show that only a quarter of the HIV counselling service providers offer 'good' quality of HIV counseling. The findings show general poor adherence to the HCT guidelines and sub-optimal content of counseling information provided to the clients, as well as inadequate amenities at the health facilities. It is important to note that poor quality of HIV counselling services reduces the effectiveness of HIV/AIDS programs, resulting into poor adherence, inadequate counselling information given and resistance to behavior change in some cases [29]. The poor quality of counselling could be attributed to the inadequate support supervision (both in content and rigor) given to the counselors coupled with counselor burnout [30-32].

Quality of HIV counselling can also be compromised by shortage of trained counselors [31]. By and large, our study established that health workers poorly adhered to the HCT guidelines. Studies conducted in South Africa including a systematic review on the use of lay counsellors in South Africa established low adherence to the HCT guidelines [33,34]. Similarly, our study established that the facilities lacked private space for counselling, and majority (66\%) of them did not follow the counselling guidelines which is comparable to a study by Evan and Ndirangu where similar findings were 
revealed [35]. Our study also revealed that majority of the health workers $(66.2 \%)$ reported that confidentiality was compromised during counselling which could be attributed to lack of private space for counselling which is comparable to similar studies [3639].

Notably, since majority of the health workers offering counselling services were registered midwives (63.5\%), poor quality of HIV counselling could have been due to understaffing [40] and also having multiple roles besides counselling which constrained counselling time [41]. Other studies also blame poor content of counselling information on shortage of health workers $[42,43]$ and insufficient knowledge [44]. However, we acknowledge that although the general content of counselling information was poor, knowledge about HIV and its transmission, information on transmission risk to the infant, adherence to treatment and that on the role of ARVs and its regimen was satisfactory, which was also in agreement with findings from a study done in Kenya that assessed male involvement in PMTCT programs [45]. Similar findings were reported by a study done in Ethiopia, where most of the surveyed health facilities only provided rudimentary information hastily, that didn't enable the clients to make informed decisions/consent [31]. Additionally, they found that the discussions between the counselor and their clients lacked depth and coverage in many of the counseling sessions, which was also consistent with what our study found. This argument is shared by another study done in Uganda that found the few available counsellors also offering a wide range of other healthcare services which subsequently affected the quality of counselling services [46]. In addition, counselling services are sometimes rushed to accommodate competing services as well as the large numbers of clients [47].

We also found the quality of content of counselling information to be poor. With regard to some of the components used to measure pre and post-test counselling, the study found that information on window period was given haphazardly as well as that on family planning and disclosure. This finding is in agreement with a study conducted in Nigeria which established that only $10 \%$ of the clients received information regarding family planning [48]. This could have been due to the fact that some of the health workers (14\%) did not have training in HIV counselling which might have similarly contributed to the sub-optimal content of counselling information delivered to the clients. We can also intuitively argue that this poor quality of content of the counselling information given by the health workers may be explained by the fact that majority (66.2\%) do not follow HIV counselling guidelines when carrying out counselling as they may miss out on delivering certain aspects on the topic to the clients. Given the poor quality of content of counselling information, the health facilities in coordination with the Ministry of Health need provide health workers with HIV counselling guidelines to follow during the counselling sessions. The health facility administrators can additionally organise HIV training sessions for counsellors who are not trained in HIV counselling.

Our findings show that there were inadequate amenities offered at majority of the health facilities studied. A similar study done in Pakistan found out that most health facilities had inadequate furniture for clients in the waiting area, inadequate counselling rooms, rest rooms and non-clean washrooms [49]. This can be explained by the fact that health facilities receive many clients (more than thirty one) for counselling per day amid the little waiting area space. Therefore, we may not only attribute the inadequacy of furniture to the huge number of mothers received, but also to the limited waiting space; which sometimes serves as the reception [40]. Similar studies conducted in public health facilities in Uganda revealed that many services such as examination, dispensing drugs and others all operate in a single room $[50,51]$. This was attributed to lack of initial planning of services to be offered by the health facility [52].

\section{Study Limitations}

The study was cross sectional which only gave a snapshot of the quality of HIV counselling in the public health facilities. There was a likelihood of social desirability bias since the study relied on the responses from the health workers. However, this was overcome by combining the three dimensions used in the study to create a composite score for evaluation of overall quality of HIV counselling services.

\section{Conclusion}

Overall, the study found that the quality of HIV counselling offered in public health facilities was poor; and that there was poor adherence to the HCT guidelines and poor content of counselling information provided to the clients. Amenities in health facilities were largely found to be inadequate. The study also highlights that providing private space for counselling is inevitable for delivery of quality counselling services to the clients. An in-depth qualita- 
tive exploration of the provider's challenges to offering quality HIV counselling is highly recommended, as well as also investigating the client's perspective of the quality of counselling they receive.

\section{Availability of Data and Materials}

All the data generated or analyzed during the current study are available from the corresponding author on reasonable request.

\section{Consent for Publication}

Not applicable.

\section{Competing Interests}

The authors declare that they have no competing interests.

\section{Funding}

The funding for this study was availed by the German government through the German Academic Exchange Service; DAAD scholarship.

\section{Acknowledgements}

The authors sincerely thank the study respondents, the administrators and health workers of the different health facilities that participated in this study.

\section{Bibliography}

1. UNAIDS: Joint United Nations Programme on AIDS: UNAIDS World AIDS Day Report. In. Geneva; (2011).

2. Matovu JK and Makumbi FE. "Expanding access to voluntary HIV counselling and testing in sub-Saharan Africa: alternative approaches for improving uptake, 2001-2007". Tropical Medicine and International Health 12.11 (2007): 1315-1322.

3. Bunnell R., et al. "Changes in sexual behavior and risk of HIV transmission after antiretroviral therapy and prevention interventions in rural Uganda". Aids 20.1 (2006): 85-92.

4. Wolff B., et al. "Evaluation of a home-based voluntary counselling and testing intervention in rural Uganda". Health Policy and Planning 20.2 (2005): 109-116.

5. Were WA., et al. "Undiagnosed HIV infection and couple HIV discordance among household members of HIV-infected people receiving antiretroviral therapy in Uganda". JAIDS Journal of Acquired Immune Deficiency Syndromes 43.1 (2006): 91-95.
6. Wanyenze RK., et al. "Acceptability of routine HIV counselling and testing, and HIV seroprevalence in Ugandan hospitals". Bulletin of the World Health Organization 86.4 (2008): 302309.

7. Perez F., et al. "Acceptability of routine HIV testing (" opt-out") in antenatal services in two rural districts of Zimbabwe". JAIDS Journal of Acquired Immune Deficiency Syndromes 41.4 (2006): 514-520.

8. Chandisarewa W., et al. "Routine offer of antenatal HIV testing (" opt-out" approach) to prevent mother-to-child transmission of HIV in urban Zimbabwe". Bulletin of the World Health Organization 85.11 (2007): 843-850.

9. Mugore L., et al. "An assessment of the understanding of the offer of routine HIV testing among pregnant women in rural Zimbabwe 1". AIDS Care 20.6 (2008): 660-666.

10. Leon N., et al. "The impact of provider-initiated (opt-out) HIV testing and counseling of patients with sexually transmitted infection in Cape Town, South Africa: a controlled trial". Implementation Science 5.8 (2010).

11. Larsson EC., et al. "Opt-out HIV testing during antenatal care: experiences of pregnant women in rural Uganda". Health Policy and Planning 27.1 (2012): 69-75.

12. Kennedy CE., et al. "Provider-initiated HIV testing and counseling in low-and middle-income countries: a systematic review". AIDS and Behavior 17.5 (2013): 1571-1590.

13. Sherr L., et al. "Voluntary counselling and testing: uptake, impact on sexual behaviour, and HIV incidence in a rural Zimbabwean cohort". Aids 21.7 (2007): 851-860.

14. Wringe A., et al. "Uptake of HIV voluntary counselling and testing services in rural Tanzania: implications for effective HIV prevention and equitable access to treatment". Tropical Medicine and International Health 13.3 (2008): 319-327.

15. Song Y., et al. "HIV-testing behavior among young migrant men who have sex with men (MSM) in Beijing, China". AIDS Care 23.2 (2011): 179-186.

16. Mlay R., et al. "Couple counselling and testing for HIV at antenatal clinics: views from men, women and counsellors". AIDS Care 20.3 (2008): 356-360.

17. Meiberg AE., et al. "Fear of stigmatization as barrier to voluntary HIV counselling and testing in South Africa (2008). 
18. Njau B., et al. "Perceived acceptability of home-based couples voluntary HIV counseling and testing in Northern Tanzania". AIDS Care 24.4 (2012): 413-419.

19. Jürgensen M., et al. "Effects of home-based voluntary counselling and testing on HIV-related stigma: findings from a clusterrandomized trial in Zambia". Social Science and Medicine 81 (2013): 18-25.

20. Zachariah R., et al. "Task shifting in HIV/AIDS: opportunities, challenges and proposed actions for sub-Saharan Africa". Transactions of the Royal Society of Tropical Medicine and Hygiene 103.6 (2009): 549-558.

21. Delva W., et al. "Quality and quantity of antenatal HIV counselling in a PMTCT programme in Mombasa, Kenya". AIDS Care 18.3 (2006): 189-193.

22. Njeru MK., et al. "A critical assessment of the WHO responsiveness tool: lessons from voluntary HIV testing and counselling services in Kenya". BMC Health Services Research 9.1 (2009): 243.

23. Nguyen PH., et al. "Incorporating elements of social franchising in government health services improves the quality of infant and young child feeding counselling services at commune health centres in Vietnam". Health Policy and Planning 29.8 (2014): 1008-1020.

24. Ngo AD., et al. "The impact of social franchising on the use of reproductive health and family planning services at public commune health stations in Vietnam". BMC Health Services Research 10.1 (2010): 54 .

25. Mayanja: Assessment Of The Quality Of Hiv Counseling And Testing Service Delivery In Private For Profit Health Units In Kampala District (2012).

26. UNAIDS: Tools for evaluating HIV voluntary counseling and testing. UNAIDS BEST PRACTICE COLLECTION. In. Geneva (2007).

27. Reeves D., et al. "Combining multiple indicators of clinical quality: an evaluation of different analytic approaches". Medical Care 45.6 (2007): 489-496.

28. American Medical Association: Measures Development, Methodology, and Oversight Advisory Committee: Recommendations on Composite Measures". Physician Consortium for Performance Improvement (PCPI) (2010).
29. Chopra M., et al. "Preventing HIV transmission to children: quality of counselling of mothers in South Africa". Acta Paediatrica 94.3 (2005): 357-363.

30. Held M and Brann M. "Recognizing HIV/AIDS volunteers' stressors and desire for support”. AIDS Care 19.2 (2007): 212214.

31. Ismail $\mathrm{H}$ and Ali A. "Status of ANC-linked HIV counseling and testing as an intervention for PMTCT in public health facilities in Addis Ababa: quality of HIV counseling given to pregnant women for PMTCT". Ethiopian Journal of Health Development 23.3 (2009).

32. Machingura F., et al. "Evidence from participatory research on community health systems for HIV treatment and support in East and Southern Africa (2012).

33. Dewing S., et al. "It's Important to Take Your Medication Everyday Okay?" An Evaluation of Counselling by Lay Counsellors for ARV Adherence Support in the Western Cape, South Africa". AIDS and Behavior 17.1 (2013): 203-212.

34. Petersen I., et al. "Optimizing lay counsellor services for chronic care in South Africa: a qualitative systematic review". Patient Education and Counseling 95.2 (2014): 201-210.

35. Evans $\mathrm{C}$ and Ndirangu E. "The nursing implications of routine provider-initiated HIV testing and counselling in sub-Saharan Africa: a critical review of new policy guidance from WHO/ UNAIDS". International Journal of Nursing Studies 46.5 (2009): 723-731.

36. Peltzer K., et al. "Implementation of the national programme for prevention of mother-to-child transmission of HIV: A rapid assessment in Cacadu district, South Africa". African Journal of AIDS Research 9.1 (2010): 95-106.

37. Bott S., et al. "Rewards and challenges of providing HIV testing and counselling services: health worker perspectives from Burkina Faso, Kenya and Uganda". Health Policy and Planning (2014): czu100.

38. Medley AM and Kennedy CE. "Provider challenges in implementing antenatal provider-initiated HIV testing and counseling programs in Uganda". AIDS Education and Prevention 22.2 (2010): 87-99.

39. Dewing S., et al. "Behaviour change counselling for ARV adherence support within primary health care facilities in the Western Cape, South Africa". AIDS and Behavior 16.5 (2012): 1286-1294. 
40. White J. "Improving Access to Healthcare in Uganda. In (2012).

41. Dinku F AG. "Assessment of Voluntary Counseling and Testing (VCT) service quality in terms of client satisfaction: A comparative study between public and private health institutions in Addis Ababa, Ethiopia". Science Journal of Clinical Medicine 2.1 (2013): 1-7.

42. Callaghan M., et al. "A systematic review of task-shifting for HIV treatment and care in Africa". Human Resources for Health 8.1 (2010): 1.

43. Leon N., et al. "The impact of provider-initiated (opt-out) HIV testing and counseling of patients with sexually transmitted infection in Cape Town, South Africa: a controlled trial". Implementation Science 5.1 (2010): 1.

44. Alam N., et al. "Effect of single session counselling on partner referral for sexually transmitted infections management in Bangladesh". Sexually Transmitted Infections 87.1 (2011): 4651.

45. Aluisio A., et al. "Male antenatal attendance and HIV testing are associated with decreased infant HIV infection and increased HIV free survival". Journal of Acquired Immune Deficiency Syndromes 56.1 (1999): 76.

46. Mugisha E., et al. "Providing integrated voluntary counselling and testing (VCT) services in Uganda". Africa Journal of Nursing and Midwifery 12.1 (2010): 52-63.

47. Haffejee S., et al. "An assessment of counselling and support services for people living withHIV in Gauteng, South Africa: findings of a baseline study". African Journal of AIDS Research 9.4 (2010): 367-372.

48. Nig AOFF., et al. "Clients' perceptions of the quality of antenatal care". Journal of the National Medical Association 100.9 (2008): 1052.

49. Agha S. "Changes in the proportion of facility-based deliveries and related maternal health services among the poor in rural Jhang, Pakistan: results from a demand-side financing intervention". International Journal for Equity in Health 10.1 (2011): 1 .

50. Healthcare a major challenge for Uganda (2016).

51. Okwero P., et al. "Fiscal Space for Health in Uganda-World Bank Working Paper No. 186. Washington, DC: The International Bank for Reconstruction and Development/The World Bank, Available: world bank (2011).
52. Konde-Lule J., et al. "Private and public health care in rural areas of Uganda". BMC International Health and Human Rights 10.1 (2010): 1.

\section{Assets from publication with us}

- Prompt Acknowledgement after receiving the article

- Thorough Double blinded peer review

- Rapid Publication

- Issue of Publication Certificate

- High visibility of your Published work

Website: www.actascientific.com/

Submit Article: www.actascientific.com/submission.php

Email us: editor@actascientific.com

Contact us: +919182824667 